\title{
Composite core-shell microparticles from microfluidics for synergistic drug delivery
}

\author{
Yanna $\mathrm{Li}^{1 \dagger}$, Dan $\mathrm{Yan}^{2 \dagger}$, Fanfan Fu${ }^{1}$, Yuxiao Liu ${ }^{1}$, Bin Zhang ${ }^{2}$, Jie Wang ${ }^{1}$, Luoran Shang ${ }^{1}$, Zhongze Gu ${ }^{1}$ \\ and Yuanjin Zhao ${ }^{1^{*}}$
}

\begin{abstract}
Microparticles have a demonstrated value for drug delivery systems. The attempts to develop this technology focus on the generation of featured microparticles for improving the function of the systems. Here, we present a new type of microparticles with gelatin methacrylate (GelMa) cores and poly(L-lactide-co-glycolide) (PLGA) shells for synergistic and sustained drug delivery applications. The microparticles were fabricated by using GelMa aqueous solution and PLGA oil solution as the raw materials of the microfluidic double emulsion templates, in which hydrophilic and hydrophobic actives, such as doxorubicin hydrochloride (DOX, hydrophilic) and camptothecine (CPT, hydrophobic), could be loaded respectively. As the inner cores were polymerized in the microfluidics when the double emulsions were formed, the hydrophilic actives could be trapped in the cores with high efficiency, and the rupture or fusion of the cores could be avoided during the solidification of the microparticle shells with other actives. The size and component of the microparticles can be easily and precisely adjusted by manipulating the flow solutions during the microfluidic emulsification. Because of the solid structure of the resultant microparticles, the encapsulated actives were released from the delivery systems only with the degradation of the biopolymer layers, and thus the burst release of the actives was avoided. These features of the microparticles make them ideal for drug delivery applications.
\end{abstract}

Keywords: microfluidic, emulsion, microparticle, drug delivery, biomaterial

\section{INTRODUCTION}

Microparticles have been extensively studied as delivery vehicles of proteins, peptides, and some small molecule drugs [1-6]. Compared with conventional forms of drug delivery via long-term frequent oral intake or injections to maintain a constant drug concentration, microparticle-based delivery systems can control both the level of the drugs and their lifetime in the body, and improve pharmacokinetics by sustained release of the drugs. Thus, the disadvantages of potential overdose upon administration and patient inconvenience during the drug delivery can be effectively avoided [7-10]. In addition, microparticle-based systems are able to deliver drugs at the site of interest to avoid biological and/or metabolic barriers, and this can lead to higher efficiency of drug delivery, lower doses, and reduced side effects $[11,12]$. These microparticle-based drug delivery systems are composed of a biodegradable polymer matrix encapsulating therapeutic agents [13-15], that can be fabricated by a variety of physical and chemical methods, including phase separation, spray drying, emulsifying solvent evaporation [16], etc. However, these microparticles usually have a broad size distribution, a great diversity in structure and a high initial burst release, all of which can reduce the drug delivery efficiency [17]. Furthermore, because of the simple structure and content of the microparticles, synergistic delivery of two or more drugs, in particular the hydrophobic and hydrophilic agents, is difficult [18-20]. The multidrug delivery systems are a promising strategy to overcome drug resistance, minimize potential toxicities by countering biological compensation, and allow reduced dosage of each agent $[21,22]$. Therefore, microparticles with controlled size, structure and content are needed for the development of efficient drug delivery systems [23-25].

Because of their capability of generating monodisperse emulsions and executing precise control over the suspended droplets inside the microchannels and their

\footnotetext{
${ }^{1}$ State Key Laboratory of Bioelectronics, School of Biological Science and Medical Engineering, Southeast University, Nanjing 210096, China

${ }^{2}$ Department of Pharmacy, Jiangsu Cancer Hospital, Nanjing 210009, China

The first two authors contributed equally to this work.

* Corresponding author (email: yjzhao@seu.edu.cn)
} 
operation, microfluidic emulsification technologies have become a powerful tool for controllable fabrication of microparticles with desired properties [26-28]. The degree of control afforded by microfluidics is highlighted by the ability to generate controlled multiple emulsions of double, triple, and an even higher-order, where the size and number of encapsulated droplets can be manipulated with high accuracy [29]. Based on the microfluidic technologies, varieties of hydrophobic and hydrophilic active ingredients can be simultaneously encapsulated into the double emulsions, which are further solidified to form stable polymers or microcapsules for the synergistic drug delivery systems [30-35]. However, because of the thermodynamic instability, these double emulsions tend to rupture during their solidification, and this reduces the encapsulation efficiency of the actives to the inner cores. In addition, most of the resultant delivery systems have a structure of liquid cores surrounded by a solid shell, which can burst release once the shell breaks or degrades. These disadvantages have limited the practical applications of the microfluidic emulsified microparticles for drug delivery. Thus, new microparticles with high encapsulation efficiency and controllable release rates are still anticipated.

In this paper, we present a new type of solid core-shell microparticle with the desired features for delivery systems, as schemed in Fig. 1. The microparticles were fabricated using two distinct biopolymer solutions as the raw materials of the microfluidic double emulsion templates, in which hydrophilic and hydrophobic actives could be loaded, respectively. As the inner cores were polymerized in the microfluidics during the formation of the double emulsion, the solute actives could be trapped in the cores with high efficiency, and the rupture or fusion of the cores could be avoided during the solidification of the microparticle shells with other actives. Because of the solid structure of the microparticles, the encapsulated actives were released from the delivery systems only with the degradation of the biopolymer layers, and thus the burst release of the actives was avoided. These features make the solid core-shell microparticles ideal for synergistic and sustained drug delivery applications.

\section{EXPERIMENTAL SECTION}

\section{Materials}

The surfactant poly(vinyl alcohol) (PVA, $87 \%-89 \%$ hydrolyzed, $\left.M_{\mathrm{w}}=13,000-23,000 \mathrm{Da}\right)$, gelatin porcine, photointiator 2-hydroxy-2-methylphenyl propanone (HMPP), doxorubicin (DOX) hydrochloride, and camptothecine (CPT) were purchased from Sigma-Aldrich. Photocurable gelatin methacrylate (GelMa, a hydrogel with high biocompatibility and biodegradability for drug or cell encapsulation) and phosphate buffered saline (PBS) were prepared in our lab. In order to achieve a better mixing, HMPP was firstly premixed with equal volume of ethanol. Then the mixture was added to a GelMa solution, with volume fraction of $1 \%\left(V_{\mathrm{HMPP}} / V_{\mathrm{GelMa}}\right)$. Poly(L-lactide-co-
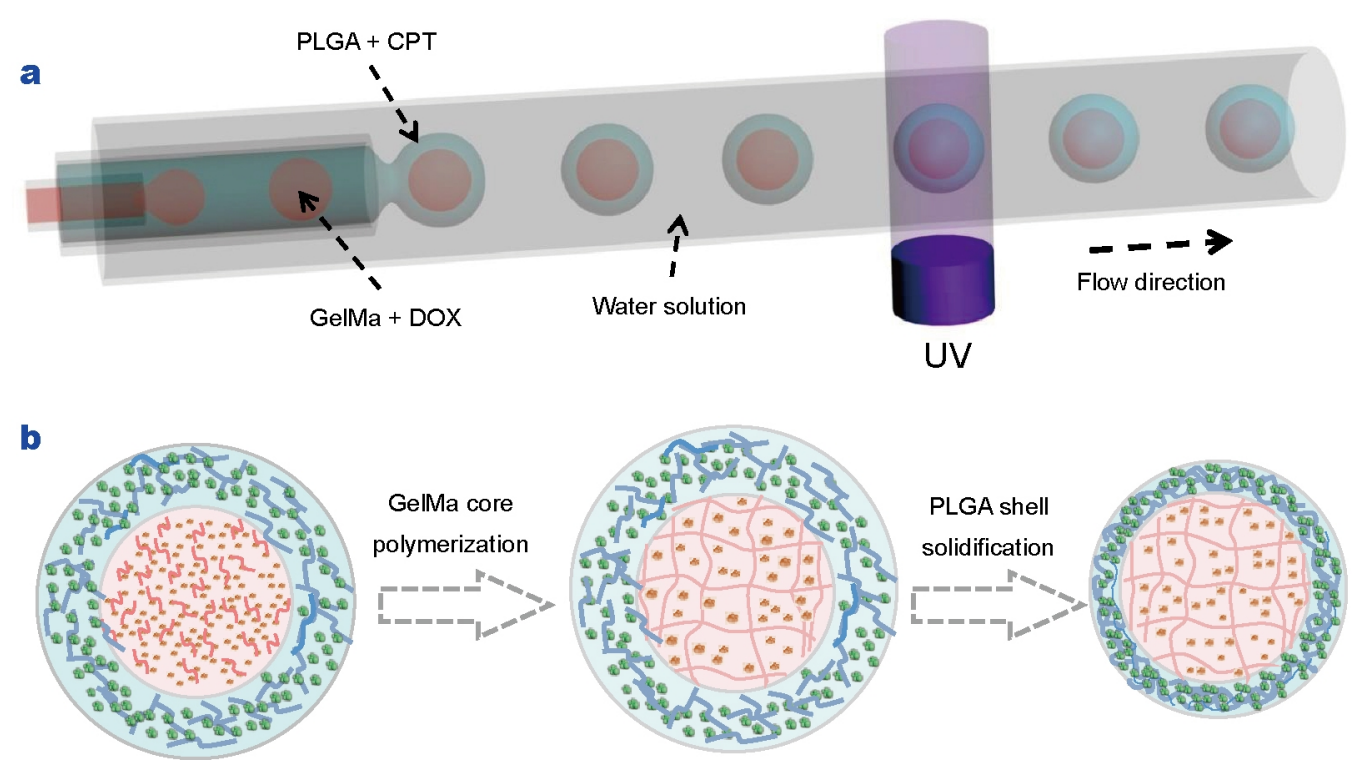

Figure 1 (a) Schematic diagram of a capillary microfluidic system for generating the W/O/W double emulsion templates with polymerized cores; (b) schematic diagram of the fabrication process of the drug loaded GelMa-PLGA core-shell microparticles. 
glycolide) (PLGA, $M_{\mathrm{w}}=7000-17,000$, lactic acid:glycolic acid=50:50) was purchased from Jinan Daigang Bio-technology Co. (China). Dichloromethane (DCM) was purchased from Sinopharm Chemical Reagent Co., Ltd. (Shanghai, China). Deionized water was used in all experiments. All other chemical reagents were of the best grade available and used as received.

\section{Preparation of drug loaded GelMa-PLGA core-shell microparticles}

One capillary microfluidic device is composed of three cylindrical glass capillary tubes (one inner, one middle and one outer) nested within a square glass tube. All the round and square glass capillary tubes were purchased from World Precision Instruments, Inc. The inner capillary was tapered by using a laboratory portable Bunsen burner (Honest MicroTorch) to reach an orifice diameter of 30-50 $\mu \mathrm{m}$. The middle capillary was tapered by a micropipette puller (Sutter Instrument Co., Novato, USA) and was sanded under optical microscope to reach the desired orifice diameter of about $100 \mu \mathrm{m}$. All of the tubes were set in good alignment to form a coaxial geometry because the outer diameter of the round tube is the same as the inner diameter of the square tube. As illustrated in Fig. 1a, the inner phase consisted of deionized water with $15 \%(w / v)$ photo-crosslinkable GelMa, in which the drug of DOX hydrochloride $\left(500 \mu \mathrm{g} \mathrm{mL}^{-1}\right)$ was dissolved. The middle oil phase was composed of $10 \%(w / v)$ PLGA and CPT (500 $\mu \mathrm{g} \mathrm{mL}^{-1}$ ) dissolved in DCM. The outermost aqueous phase was deionized water with $2 \%(w / v)$ PVA. The inner and middle phases flowed in the same direction via the injection capillaries and the outer phase flowed via the interstices between the square tube and the collection tube. Each fluid was connected with a glass syringe (SGE Analytical Science) through a polyethylene tube (Scientific Commodities Inc.), and was pumped into the entrance of each tube by a syringe pump (Harvard PHD 2000 Series). The double emulsion generation process in the collection tube of the device could be observed in real time with a stereomicroscope (NOVEL NTB-3A, Ningbo Yongxin Optics Co., Ltd, China) and was recorded by a charged coupled device (CCD, Media Cybernetics Evolution MP 5.0 RTV). The polymerization of the GelMa cores of the double emulsion was induced by UV-light (EXFO OmniCure SERIES 1000, $365 \mathrm{~nm}, 100 \mathrm{~W}$ ) in the collection channel. The UV light intensity is very weak, and the irradiation time is less than $20 \mathrm{sec}$. Then the emulsion droplets were collected with $2 \%(w / v)$ aqueous PVA solution in a glass flask, and the DCM was evaporated under reduced pressure at room temperature for $\sim 24 \mathrm{~h}$ by using a rotary evaporator (RV10, IKA-WERKE, German) so that the shells were hardened. The obtained microparticles were washed with excess amount of deionized water and then dried.

\section{Characteristics of drug loaded GelMa-PLGA core-shell microparticles}

The outer radius $\left(R_{\text {outer }}\right)$, inner radius $\left(R_{\text {inner }}\right)$, and shell thickness of the core-shell microparticles were all measured by using an AOS Imaging Studio V3.4.2 software. The shell thickness was calculated by $R_{\text {outer }}-R_{\text {inner. }}$ The colored optical images of the microparticles were observed by a stereomicroscope (Nikon SMZ 745T) and were captured by the same CCD as mentioned above. The morphology and the drug distribution of the fabricated core-shell microparticles were characterized using a confocal laser scanning microscope (CLSM, FV10i, OLYMPUS, Japan). In order to obtain the cross-section, the microparticle was cut from the middle by using a sharp blade after freeze-drying. Scanning electron microscope (SEM, Hitachi S3000N) images were obtained after coating a thin layer of gold on the microparticles.

\section{Determination of drug content and encapsulation efficiency} The content of CPT was determined by lysing a certain amount of microparticles in $1 \mathrm{~mL}$ of dimethyl sulfoxide (DMSO). The concentration of CPT in the supernatant was assayed by the UV method at 365 and $570 \mathrm{~nm}$ (reference) with a microplate reader (SYNERGY|HTX). For the content of DOX, the unloaded DOX content in the solution was detected by the UV method at $499 \mathrm{~nm}$ with the same microplate reader, and then subtracted by the total amount used. The drug content of the microparticles was defined as the weight percentage of CPT and DOX loaded in GelMaPLGA microparticles. The drug loading content could be further increased by adding more drugs in the pre-gel solution. The encapsulation efficiency was defined as the ratio of the actual amount of encapsulated CPT and DOX over the total amount of CPT and DOX used, respectively.

\section{Measurement of in vitro drug release}

A certain amount of DOX-CPT-loaded GelMa-PLGA coreshell microparticles were dispersed in $1 \mathrm{~mL}$ of PBS (PH 7.4) in centrifuge tubes and oscillating incubated at $37^{\circ} \mathrm{C}$. At every predetermined time interval, the samples were centrifuged and $200 \mu \mathrm{L}$ of the supernatants were removed to another centrifuge tube and then filtered and assayed by using a microplate reader. An equivalent volume $(200 \mu \mathrm{L})$ of fresh PBS was added to each tube at each time point after 
assaying. During the first $24 \mathrm{~h}$, the supernatants were taken every $1 \mathrm{~h}$, and after that were measured every $2 \mathrm{~d}$. The unloaded GelMa-PLGA core-shell microspheres were used as control.

\section{Synergistic drugs to tumor cells}

The advantages of oncotherapy of the synergistic drug delivery system were assessed by analyzing cell viability treated with the fabricated microparticles via the live calcein-green fluorescence and the MTT (3-(4,5-dimethylfthiazol-2-yl)-2,5-diphenyl tetrazolium bromide) assay. Human hepatoma (HepG2) cell lines and human colon carcinoma (HCT116) cell lines were investigated as model tumor cell lines. HCT116 cells were seeded in 24-well with $2 \mathrm{~mL}$ Dulbecco's modified eagle medium (DMEM) per well. After incubating for $24 \mathrm{~h}$, the wells were separated into eight groups with three wells for each group. The different groups of wells were added PBS as control, only free DOX $\left(5 \mu \mathrm{g} \mathrm{mL} \mathrm{m}^{-1}\right)$, only free CPT $\left(5 \mu \mathrm{g} \mathrm{mL} \mathrm{m}^{-1}\right)$, free DOX $\left(5 \mu \mathrm{g} \mathrm{mL}^{-1}\right)$ and CPT $\left(5 \mu \mathrm{g} \mathrm{mL} \mathrm{m}^{-1}\right)$, unloaded microparticles $\left(500 \mu \mathrm{g} \mathrm{mL}^{-1}\right)$, DOX-loaded microparticles (500 $\left.\mu \mathrm{g} \mathrm{mL}^{-1}\right)$, CPT-loaded microparticles $\left(500 \mu \mathrm{g} \mathrm{mL}^{-1}\right)$, and DOX-CPT co-loaded microparticles $\left(500 \mu \mathrm{gL}^{-1}\right)$, respectively.

HepG2 cells were seeded in 12-well plates with $3 \mathrm{~mL}$ DMEM per well for $24 \mathrm{~h}$. Four groups of wells (three wells in each group) were added unloaded microparticles $\left(500 \mu \mathrm{g} \mathrm{mL}^{-1}\right)$, DOX-loaded microparticles $\left(500 \mu \mathrm{g} \mathrm{mL}^{-1}\right)$, CPT-loaded microparticles $\left(500 \mu \mathrm{g} \mathrm{mL}^{-1}\right)$, and DOX-CPT co-loaded microparticles $\left(500 \mu \mathrm{g} \mathrm{mL}^{-1}\right)$, respectively. In brief, GeLMa-PLGA core-shell microparticles were put in each well as described above, and then incubated with the tumor cells at $37^{\circ} \mathrm{C}$ for $24 \mathrm{~h}$. They were used for synergistic drug delivery in cxcellular region of DMEM. After $24 \mathrm{~h}$ of incubation, the cell configurations were observed using the optical microscope and fluorescence microscope and the cell viability was evaluated by MTT assay.

Phase contrast photomicrographs of HCT116 cells treated with different groups of microparticles were obtained at $0,3,7,12,24$ and $36 \mathrm{~h}$. For the MTT assay, MTT was dissolved in PBS at $1 \mathrm{mg} \mathrm{ml} \mathrm{m}^{-1}$ and filtered through a $0.2 \mu \mathrm{m}$ membrane. The MTT $\left(0.1 \mathrm{mg} \mathrm{mL} \mathrm{mL}^{-1}\right)$ stock was added into each well, and the plates were wrapped in tin foil and incubated at $37^{\circ} \mathrm{C}$ for another $4 \mathrm{~h}$. Then the medium in each well was carefully removed, and the purple formazan products were dissolved with $1 \mathrm{~mL}$ of DMSO. A $100 \mu \mathrm{L}$ solution was extracted from each well and transferred to a 96-well plate and assayed at wavelengths of 570 and $690 \mathrm{~nm}$ (reference). The relative cell viability (\%) related to the control wells was calculated by $[\text { Absorbance }]_{\text {test }} /[\text { Absorbance }]_{\text {control }} \times 100$. Cell viability was also assessed by calcein-AM staining. The cultured HCT116 cells were incubated with calcein AM $\left(2 \mu \mathrm{g} \mathrm{mL}^{-1}\right)$ for $15 \mathrm{~min}$ at $37^{\circ} \mathrm{C}$. After that, the cells were rinsed by PBS, and the fluorescent images were obtained using a Nikon inverted microscope (Nikon Eclipse TE200).

\section{RESULTS AND DISCUSSION}

In this study, we used a microfluidic device that generated monodisperse water-in-oil-in-water (W/O/W) double emulsion templates in a single step. It allowed precise control of both the outer and inner drop sizes, as well as the number of droplets encapsulated in each larger drop. Our device was composed of three cylindrical glass capillary tubes nested within a square glass tube. We achieved good alignment to form a total coaxial geometry by ensuring that the outer diameter of the round tubes was the same as the inner lattice of the square tube. The generation processes of the solid core-shell microparticles by the microfluidic device are schematically depicted in Fig. 1. The inner phase consisted of deionized water with 15\% $(w / v)$ photo-crosslinkable GelMa, in which the drug of DOX hydrochloride was dissolved. The middle oil phase was composed of $10 \%(w / v)$ PLGA and CPT dissolved in DCM. The outermost aqueous phase was deionized water with $2 \%(w / v)$ PVA). When these fluids flowed through the corresponding capillaries, aqueous GelMa core droplets were generated at the end of the inner capillary and then encapsulated by a shell drop of the PLGA at the end of the middle capillary (Fig. 2a-c). As the GelMa used in this study is a kind of photopolymer, the generated emulsion templates could be photopolymerized under UV illumination downstream from the fluidic channel, producing stable double emulsions with hydrogel cores and DCM shells with dissolved PLGA. The complete solid core-shell microparticles were achieved by evaporating the DCM solvent of the shell under reduced pressure using a rotary evaporator with an addition of extra surfactant solution (Fig. 1b).

The overall size of the solid microparticles and the number of the encapsulated GelMa core droplets could be adjusted by using different orifice sizes or tuning the velocities of the three phases (see Supplementary information, Fig. S1). To achieve microparticles with stable core-shell structures for encapsulation of the actives, we should fabricate the double emulsion templates with full encapsulation of the aqueous core droplets. Thus, the ratio of the middle PLGA flowrate to the inner aqueous GelMa flowrate could be maintained much higher than the ratio of the shell to 

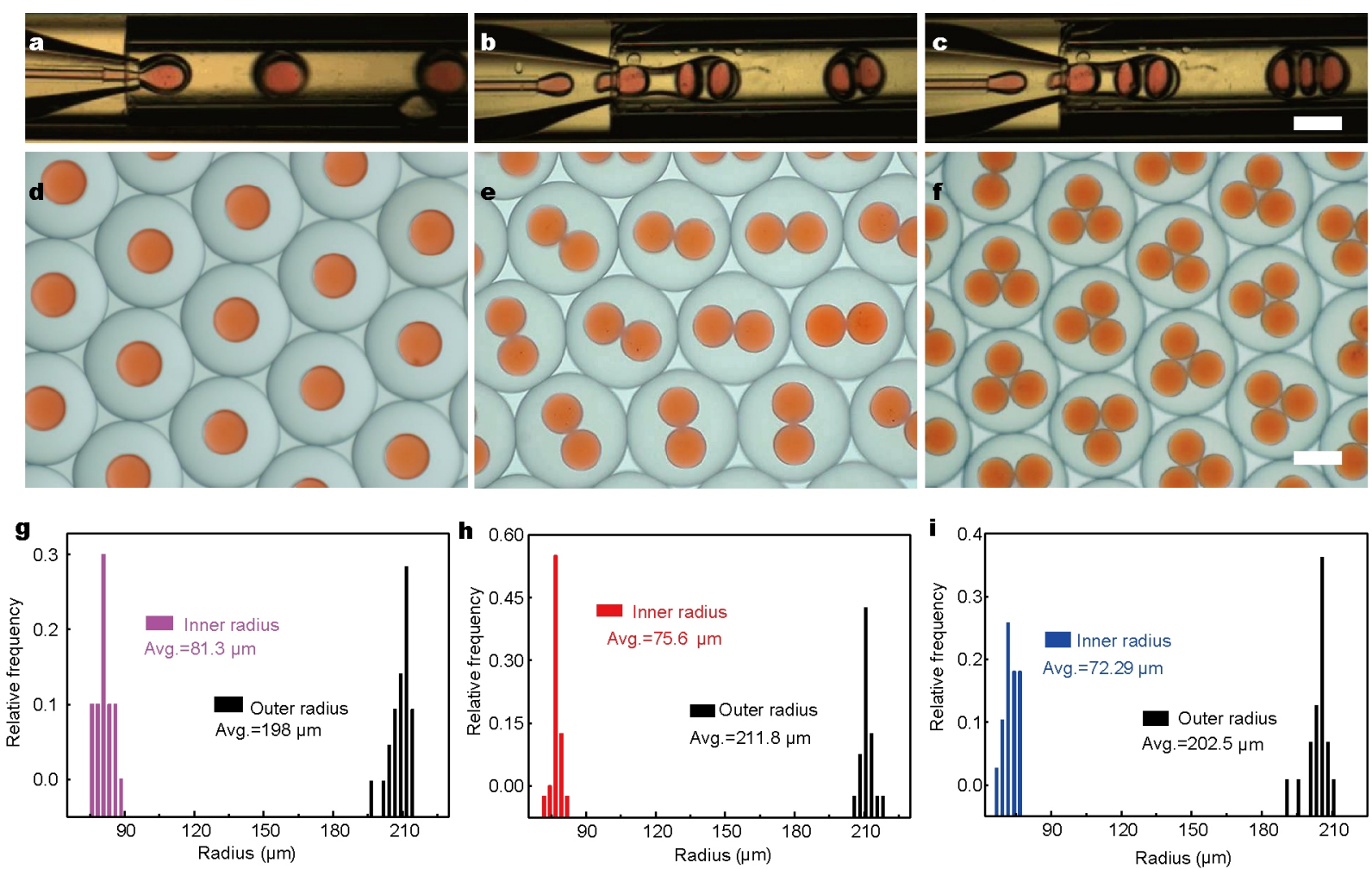

Figure 2 (a-c) Real-time microscopic images of the microfluidic generation process of the W/O/W double emulsion templates encapsulated with tunable number of cores. The scale bar is $100 \mu \mathrm{m}$; $(\mathrm{d}-\mathrm{f})$ optical microscope image of the monodisperse core-shell double emulsions with one, two and three cores, respectively. The scale bar is $200 \mu \mathrm{m}$; (g-i) the size distributions of the inner radiuses and outer radiuses of the double emulsions with one, two and three cores, respectively.

core volume in the critical packing state. It is worth mentioning that the thickness of the PLGA shells could also be tailored by using a different concentration of PLGA solution for the double emulsion generation (Fig. S2).

With our method, the aqueous drugs were locked in the GelMa hydrogel with high efficiency and encapsulated into the cores of the microparticles instantaneously when their double emulsion templates were formed. These templates with the GelMa hydrogel cores have high stability, as shown in Fig. 2d-f. Both their inner GelMa hydrogel cores and DCM shells with dissolved PLGA are highly uniform, as indicated by the statistics in Fig. 2g-i. The synergistic drug-loaded GelMa-PLGA core-shell microparticles (GelMa-PLGA-DOX-CPT) were obtained by further solidifying the shell DCM solvent of the GelMa-PLGA double emulsion droplets. The microparticles could be filtered from the continue phase and dried for preserving after they solidified. This ensures long-term stability of the microparticles and good encapsulation of the synergistic drugs.

As the double emulsion templates have solid hydrogel cores, the rupture or fusion of the cores can be avoided dur- ing the solidification of the microparticle shells with CPT. Thus, PLGA microparticles with multiple GelMa hydrogel cores can be achieved. This structure indicates that a more complex synergistic drug combination with several kinds of actives can be loaded in our microparticles for functional delivery systems. Here, as a typical example, only two distinct drugs, hydrophilic DOX and hydrophobic CPT, were used for the following study. DOX and CPT are both intrinsically fluorescent, with red and blue fluorescence, respectively, which allows us to monitor the behavior of each drug in the microparticles by fluorescent microscopy. The localizations of the synergistic hydrophilic DOX and hydrophobic CPT drugs in the GelMa-PLGA core-shell microparticles were further investigated by using CLSM, as shown in Fig. 3. It is found that the GelMa hydrogel cores are well encapsulated by the PLGA shells, irrespective of the core numbers of the microparticles. In addition, both of the DOX and CPT are distributed quite uniformly throughout the GelMa hydrogel cores and PLGA shells (Fig. S3), respectively, indicating that the synergistic drugs are loaded into the microparticles as desired.

The concentration of the loaded drugs in the microparti- 


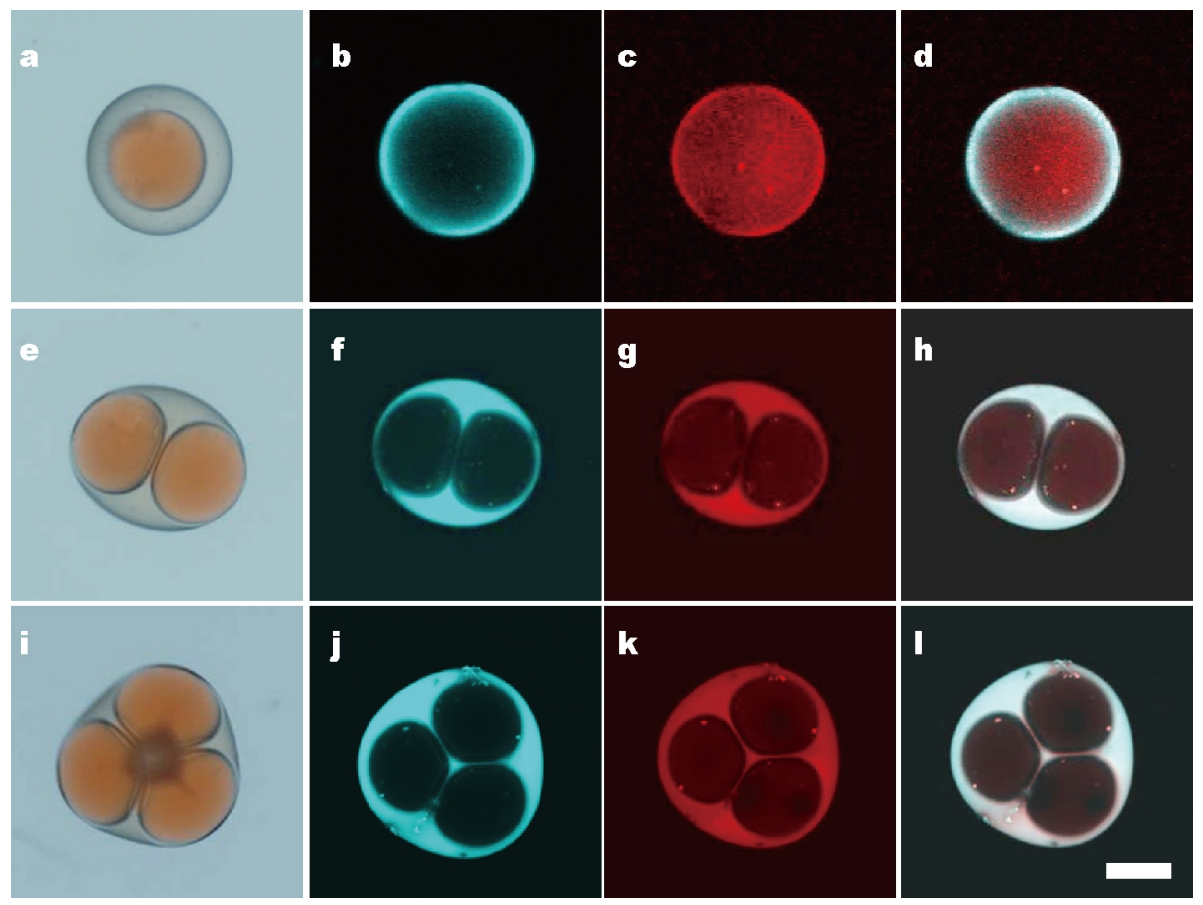

Figure 3 Optical microscopy images (a, e and i) and CLSM images (others) of the DOX and CPT drugs loaded core-shell microparticles. (a-d) Microparticle with single core; (e-h) microparticle with two cores; (i-l) microparticle with three cores. The red and blue fluorescence indicates DOX and CPT, respectively. The scale bar is $100 \mu \mathrm{m}$.

cles could be individually adjusted by dissolving them with the corresponding concentrations in the GelMa and PLGA solutions during the microfluidic emulsification. The relative ratios of the synergistic drugs could also be adjusted by controlling the structure of the double emulsion templates and the resultant microparticles. Attractively, synergistic drugs could be simultaneously encapsulated in the same individual microparticle at the desired concentrations, thus ensuring delivery of both actives at the same location for optimum synergistic activity. Moreover, the use of capillary microfluidics to generate these microparticles in a one-step process avoided the need for filling the microparticles with the drugs post-production and ensured very high encapsulation efficiency, as no excess material was generated.

The microstructures of the drug-loaded core-shell microparticles were characterized using an SEM (Fig. 4 and Fig. S4). The microparticles from a typical experiment have a size of about $210 \mu \mathrm{m}$. Close-up examination of the cross-sectioned microparticle reveals the core and shell structure of the microparticle (Fig. 4b, c). For these microparticles, the shell thickness and core diameter are about 25 and $160 \mu \mathrm{m}$, respectively. Many small pores scatter throughout the surface of the PLGA shell, which resulted from the evaporation of DCM (Fig. $4 \mathrm{~d}$, e). These pores allow the initial diffusion of the drug into the envi- ronment. The porous structures are also observed in the GelMa cores with freeze-dry treatment (Fig. 4f).

The encapsulation efficiencies of the synergistic drugs in the different GelMa-PLGA core-shell microparticles were investigated with increasing the shell thickness of the core-shell microparticles from 22 to $60 \mu \mathrm{m}$. The drug contents and encapsulation efficiencies with different thicknesses are listed in Table 1. During the fabrication process, the GelMa cores were solidified by UV light after they were generated instantly, so almost all the DOX was encapsulated in the cores. The encapsulation efficiency of the CPT in the shells was lower than that of DOX in the cores because of the diffusion of a fraction of CPT during the solvent evaporation. The formation efficiency of this core-shell structure was estimated to be at $\sim 100 \%$. These figures sustain the uniform thickness of the shell which contributes to the improvement in the drug encapsulation effectiveness and uniformity. In addition, filtering and drying caused the microparticles to exist free (Fig. S5). Thus, therapeutic application of the microparticles can be performed either via a suspension that is directly injected into the tissue or preferentially via the oral route as dried particles packed within a gelatin capsule for individual drug dosing.

As the PLGA shells and GelMA cores are biocompatible 

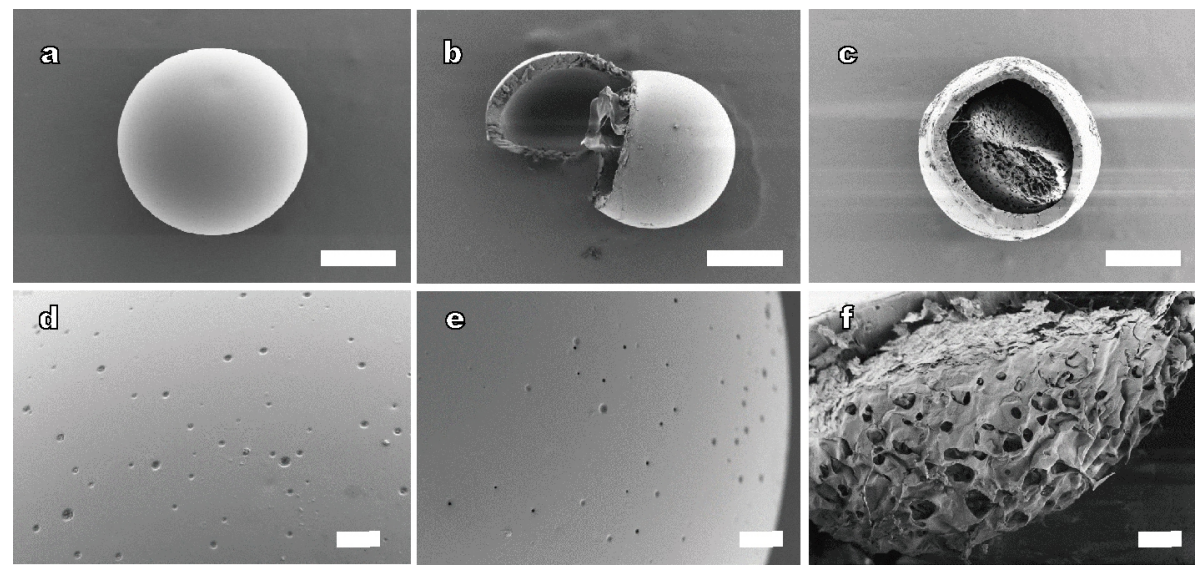

Figure 4 SEM images of the core-shell structure microparticles. (a) External view of a whole microparticle; (b) external view of a shell opened microparticle encapsulated with one core; (c) cross section image of the GelMa-PLGA core-shell microparticle; ( $\mathrm{d}$ and e) magnified images of shell surfaces of the microparticles in (a,b); (f) magnified image of a partial structure of GelMa core. The scale bars are $100 \mu \mathrm{m}$ in $(\mathrm{a}-\mathrm{c})$ and $10 \mu \mathrm{m}$ in $(\mathrm{d}-\mathrm{f})$.

Table 1 Effects of the shell thickness on loading content of drugs and encapsulation efficiency

\begin{tabular}{cccc}
\hline Shell thickness $(\mu \mathrm{m})$ & Encapsulation efficiency of CPT $(\%)$ & Encapsulation efficiency of DOX (\%) & Loading content $(\%)$ \\
\hline 22 & $45.80 \pm 2.02$ & $85.13 \pm 0.99$ & $4.06 \pm 0.02$ \\
40 & $57.27 \pm 0.89$ & $89.22 \pm 1.12$ & $6.17 \pm 0.15$ \\
60 & $60.50 \pm 1.24$ & $92.73 \pm 2.57$ & $6.88 \pm 0.24$ \\
\hline
\end{tabular}

and biodegradable both in vitro and in vivo, the microparticles can be degraded and their encapsulated actives can be released at different positions. Thus, for the application of these drug delivery core-shell microparticles, the loaded DOX was first released from the GelMa core and then diffused through the PLGA shell into the outer environment. PLGA is known to exhibit an exponential decrease in molecular weight with degradation time, so the degrading PLGA shell served as a diffusion barrier against its release from the inner region of the shell. Meanwhile, the loaded CPT was first diffused via pores throughout the PLGA shell, and with the degradation of PLGA, the drugs were released gradually (Fig. 5).

To further investigate the performance of the microparticles as delivery systems, the release kinetics of the two drugs from GelMa-PLGA-DOX-CPT microparticles was recorded (Fig. 6). Two different drugs were loaded in the core-shell microparticles of the same size $(210 \mu \mathrm{m})$ and different shell thicknesses (about 22 and $60 \mu \mathrm{m}$, respectively) were chosen for this study. The release curves of DOX and CPT from the two different microparticles are plotted in Fig. 6a-d. Due to the existence of the nanopore in the shell of the microparticles (as indicated in Fig. 4d, e), the inner encapsulated DOX can initially release out as the shell encapsulated CPT. As the recorded time is long, the plotted curves seem like a burst release at the beginning in Fig. 6a, b. However, their release is actually slow at the first $12 \mathrm{~h}$, as shown in Fig. 6c, d. The release of both CPT and DOX from the GelMa-PLGA microparticles is found to be sustained. For the microparticles with a shell thickness of $60 \mu \mathrm{m}$, the release of CPT and DOX is slower, about $51 \%$ of CPT and $22 \%$ of DOX released within $72 \mathrm{~h}$. Thus, the drugs' release profiles are more sustained and with a lower burst release for microparticles with thicker shells. It is worth mentioning that the drug release was related with the numbers of cores only when we fixed the volumes of encapsulated content. The relation between the DOX release percentage and the number of GelMa cores is recorded in Fig. S6.

To confirm the advantages of the synergistic drug delivery system for oncotherapy, the effects of the core-shell microparticles were investigated on HCT116 and HepG2 cells. DOX and CPT were paired to demonstrate the co-delivery synergistic effect of two chemotherapeutical drugs in the same vehicle. DOX can bind to DNA by intercalation and motivates a series of biochemical events including apoptosis in tumor cells, and CPT can stabilize the normally transient cleavable DNA-topo ${ }^{\mathrm{I}}$ complex and forms an enzyme-drug-DNA ternary cleavable complex [36-38]. The collision of the replication fork with a cleaved strand of DNA causes an irreversible arrest of the replication fork, 

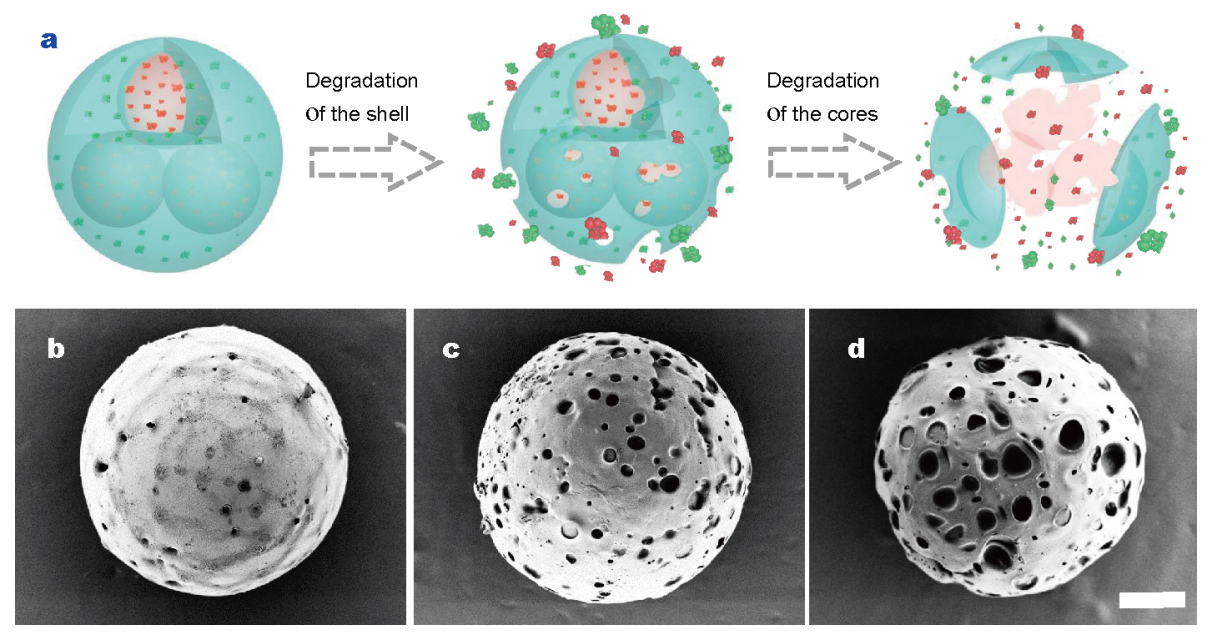

Figure 5 (a) Schematic diagram of the microparticle degradation and its drug release; (b-d) SEM images of the GelMa-PLGA core-shell microparticles during the degradation and release periods of $72,168,360 \mathrm{~h}$, respectively. The scale bar is $50 \mu \mathrm{m}$.
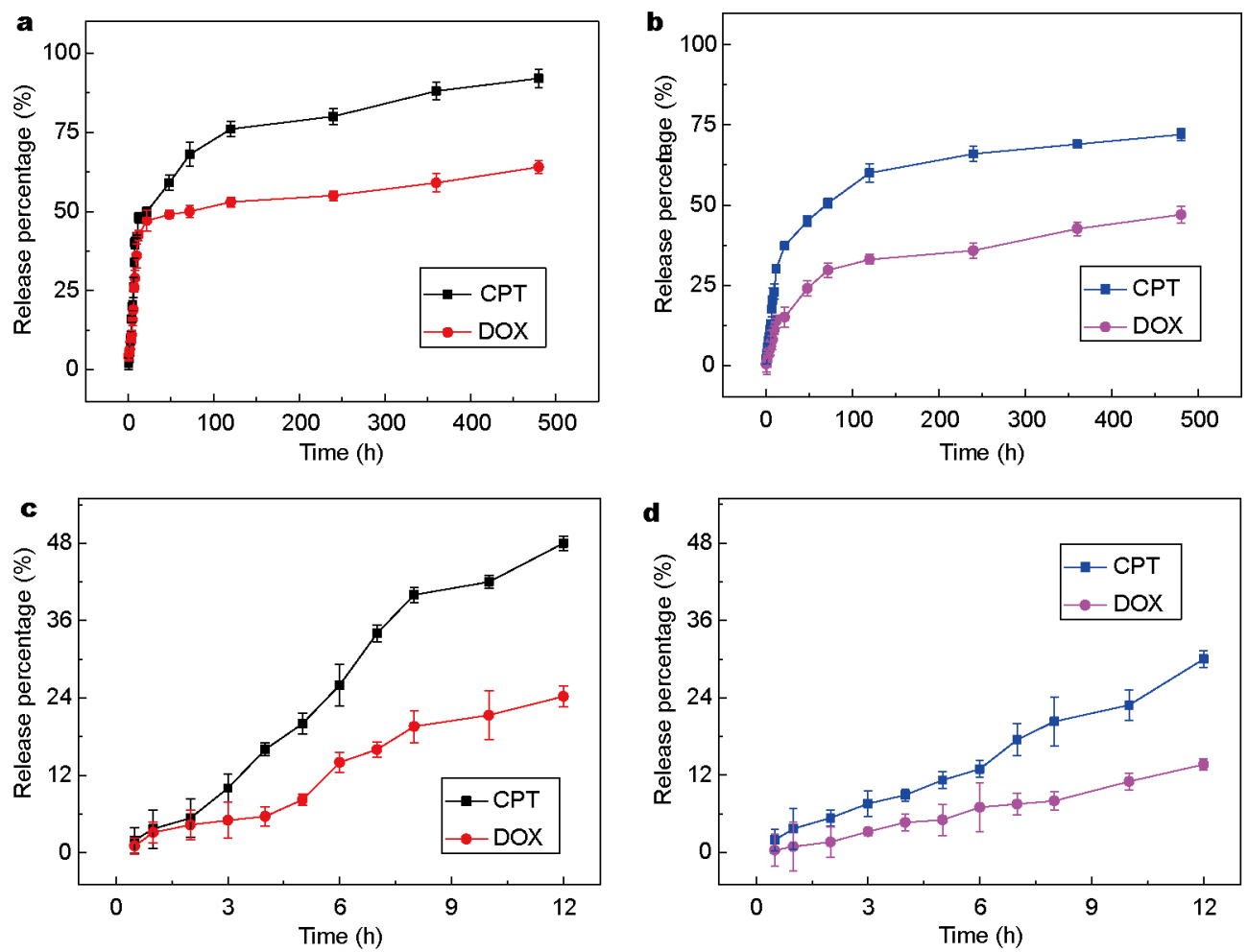

Figure 6 In vitro accumulative CPT and DOX release from GelMa-PLGA core-shell microparticles. (a, c) Drugs release from thin shell (22 $\mu \mathrm{m}) \mathrm{mi}$ croparticles; (b, d) drugs release from thick shell $(60 \mu \mathrm{m})$ microparticles. (c, d) The first $12 \mathrm{~h}$ processes of $(\mathrm{a}, \mathrm{b})$, respectively. Error bars represent standard deviations.

double-strand DNA breakage and cell death, including most of tumor cells and some normal cells. Here, the tumor cells were treated for $24 \mathrm{~h}$ with the unloaded, single DOX loaded, single CPT loaded, and both DOX and CPT loaded microparticles (shell thickness of $60 \mu \mathrm{m}$ ), respectively. The cells cultured with free DOX, CPT, and DOX/CPT solutions were also set as control groups. Representative optical and fluorescence microscopy cell culture results are presented in Fig. 7 and Figs S7, S8 (for HCT116 cells) and S9 (for HepG2 cells). It can be observed that the HCT116 cells and HepG2 cells grow well in the culture plate with the presence of microparticles unloaded with the drugs. 


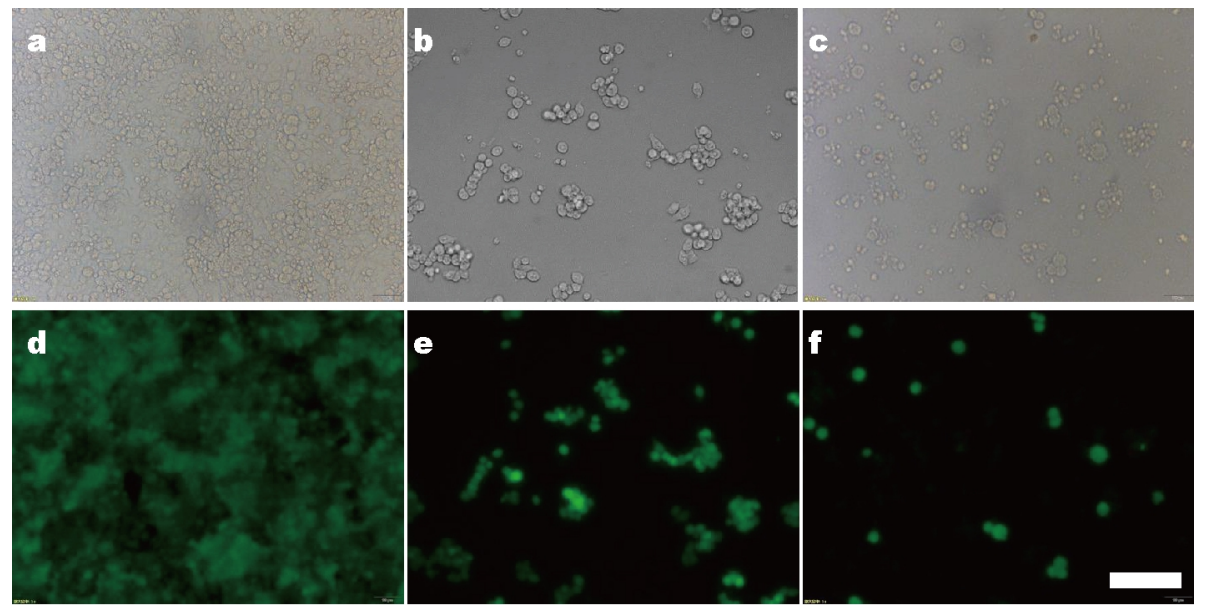

Figure 7 Optical and fluorescence microscopy images of HCT116 cells treated with unloaded microparticles (a, d), only CPT-loaded microparticles (b, e), and DOX-CPT-co-loaded microparticles (c, f) for $24 \mathrm{~h}$, respectively. The scale bar is $50 \mu \mathrm{m}$.

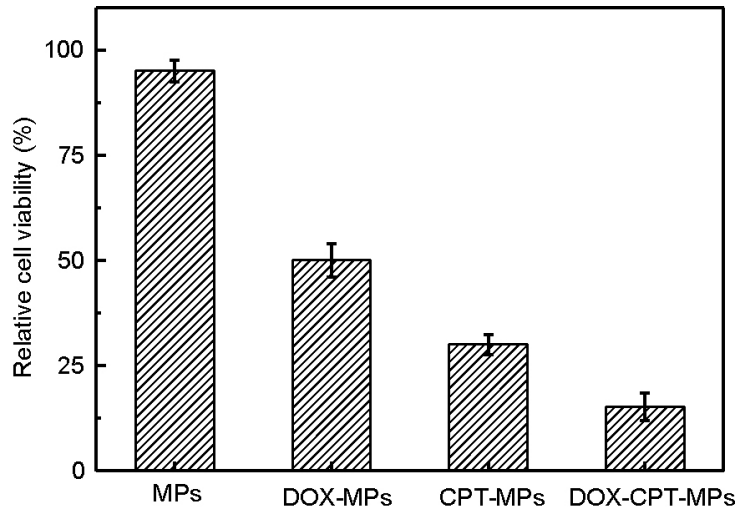

Figure 8 Result of the MTT assay of the HCT116 cells treated with unloaded microparticles (MPs), only DOX-loaded microparticles (DOX-MPs), only CPT-loaded microparticles (CPT-MPs), and DOX-CPT-co-loaded microparticles (DOX-CPT-MPs) for $24 \mathrm{~h}$. Error bars represent standard deviations.

However, when the microparticles are loaded with either DOX or CPT, more than 50\% HCT116 cells and $60 \%$ HepG2 cells are killed (Figs S8 and S9). More attractively, with the synergistic drug delivery microparticles, less than 20\% HCT116 cells (Fig. 8 and Fig. S10) and 10\% HepG2 cells (Fig. S11) survive, respectively. These effects can also be observed clearly from the morphology and population changes of the HCT116 cells during different culture time of synergistic drug delivery microparticles, as shown in Fig. S12. Thus, co-delivery of DOX and CPT in the core-shell microparticles can significantly reduce cell viability, and enhance therapeutic efficacy in treating cancer cells.

\section{CONCLUSIONS}

In conclusion, the monodisperse GelMa-PLGA core-shell microparticles with controlled core size and shell thickness were generated by using a simple capillary microfluidic method. These microparticles were loaded with hydrophilic and hydrophobic actives, such as hydrophilic DOX and hydrophobic CPT, and were employed for the application of synergistic and sustained drug delivery. As the inner GelMa cores were polymerized in the microfluidics when the double emulsion templates were formed, the hydrophilic actives could be trapped in the cores with high efficiency, and the rupture or fusion of the cores during the solidification of the microparticle shells can be avoided with hydrophobic actives. Because of the solid structure of the microparticles, the encapsulated actives were sustainably released from the delivery systems with the degradation of the biopolymer layers. It was demonstrated that the core-shell microparticles with DOX and CPT co-delivery could significantly reduce cell viability, and enhance therapeutic efficacy in treating liver cancer cells. These features of the microparticles indicate their potential value as functional delivery systems.

\section{Received 27 October 2016; accepted 13 December 2016; published online 22 January 2017}

1 Zarzar LD, Sresht V, Sletten EM, et al. Dynamically reconfigurable complex emulsions via tunable interfacial tensions. Nature, 2015, 518: $520-524$

2 Zhang H, Liu D, Shahbazi MA, et al. Fabrication of a multifunctional nano-in-micro drug delivery platform by microfluidic templated encapsulation of porous silicon in polymer matrix. Adv Mater, 2014, 26: 4497-4503

3 Min NG, Ku M, Yang J, et al. Microfluidic production of uniform microcarriers with multicompartments through phase separation in emulsion drops. Chem Mater, 2016, 28: 1430-1438

4 Rothenfluh DA, Bermudez H, O’Neil CP, et al. Biofunctional polymer nanoparticles for intra-articular targeting and retention in cartilage. Nat Mater, 2008, 7: 248-254 
5 Wang J, Shang L, Cheng Y, et al. Microfluidic generation of porous particles encapsulating spongy graphene for oil absorption. Small, 2015, 11: 3890-3895

6 Zhang L, Feng Q, Wang J, et al. Microfluidic synthesis of hybrid nanoparticles with controlled lipid layers: understanding flexibility-regulated cell-nanoparticle interaction. ACS Nano, 2015, 9: 9912-9921

7 Agnihotri SA, Mallikarjuna NN, Aminabhavi TM. Recent advances on chitosan-based micro- and nanoparticles in drug delivery. J Control Release, 2004, 100: 5-28

8 Lei Y, Hamada Y, Li J, et al. Targeted tumor delivery and controlled release of neuronal drugs with ferritin nanoparticles to regulate pancreatic cancer progression. J Control Release, 2016, 232: $131-142$

9 Zhang B, Cheng $\mathrm{Y}$, Wang $\mathrm{H}$, et al. Multifunctional inverse opal particles for drug delivery and monitoring. Nanoscale, 2015, 7: 10590-10594

10 Sivakumar S, Bansal V, Cortez C, et al. Degradable, surfactant-free, monodisperse polymer-encapsulated emulsions as anticancer drug carriers. Adv Mater, 2009, 21: 1820-1824

11 Zhang J, Tao S, Zhang B, et al. Microparticle-based strategy for controlled release of substrate for the biocatalytic preparation of L-homophenylalanine. ACS Catal, 2014, 4: 1584-1587

12 Ranganath SH, Tong Z, Levy O, et al. Controlled inhibition of the mesenchymal stromal cell pro-inflammatory secretome via microparticle engineering. Stem Cell Rep, 2016, 6: 926-939

13 Shum HC, Kim JW, Weitz DA. Microfluidic fabrication of monodisperse biocompatible and biodegradable polymersomes with controlled permeability. J Am Chem Soc, 2008, 130: 9543-9549

14 Windbergs M, Zhao Y, Heyman J, et al. Biodegradable core-shell carriers for simultaneous encapsulation of synergistic actives. J Am Chem Soc, 2013, 135: 7933-7937

15 Liu B, Wang Y, Yang F, et al. Construction of a controlled-release delivery system for pesticides using biodegradable PLA-based microcapsules. Colloids Surfaces B-Biointerfaces, 2016, 144: 38-45

16 Jain RA. The manufacturing techniques of various drug loaded biodegradable poly(lactide-co-glycolide) (PLGA) devices. Biomaterials, 2000, 21: 2475-2490

17 Zhao Y, Shum HC, Adams LLA, et al. Enhanced encapsulation of actives in self-sealing microcapsules by precipitation in capsule shells. Langmuir, 2011, 27: 13988-13991

18 Choi CH, Weitz DA, Lee CS. One step formation of controllable complex emulsions: from functional particles to simultaneous encapsulation of hydrophilic and hydrophobic agents into desired position. Adv Mater, 2013, 25: 2536-2541

19 Shum HC, Zhao Y, Kim SH, et al. Multicompartment polymersomes from double emulsions. Angew Chem Int Ed, 2011, 50: $1648-1651$

20 Liu B, Möhwald H, Wang D. Synthesis of Janus particles via kinetic control of phase separation in emulsion droplets. Chem Commun, 2013, 49: 9746-9748

21 Kim JH, Jeon TY, Choi TM, et al. Droplet microfluidics for producing functional microparticles. Langmuir, 2014, 30: 1473-1488

22 Datta SS, Abbaspourrad A, Amstad E, et al. 25th anniversary article: double emulsion templated solid microcapsules: mechanics and controlled release. Adv Mater, 2014, 26: 2205-2218

23 He C, Tang Z, Tian H, et al. Co-delivery of chemotherapeutics and proteins for synergistic therapy. Adv Drug Deliver Rev, 2016, 98: 64-76

24 Chen AM, Zhang M, Wei D, et al. Co-delivery of doxorubicin and Bcl-2 siRNA by mesoporous silica nanoparticles enhances the ef- ficacy of chemotherapy in multidrug-resistant cancer cells. Small, 2009, 5: 2673-2677

25 Zhao Y, Shum HC, Chen $\mathrm{H}$, et al. Microfluidic generation of multifunctional quantum dot barcode particles. J Am Chem Soc, 2011, 133: $8790-8793$

$26 \mathrm{Ge} \mathrm{H}, \mathrm{Xu} \mathrm{H}, \mathrm{Lu} \mathrm{T}$, et al. Microfluidic production of porous carbon spheres with tunable size and pores. J Colloid Interface Sci, 2016, 461: $168-172$

27 Zhao Y, Cheng Y, Shang L, et al. Microfluidic synthesis of barcode particles for multiplex assays. Small, 2015, 11: 151-174

28 Kim SH, Won Shim J, Lim JM, et al. Microfluidic fabrication of microparticles with structural complexity using photocurable emulsion droplets. New J Phys, 2009, 11: 075014

29 Wang J, Cheng Y, Yu Y, et al. Microfluidic generation of porous microcarriers for three-dimensional cell culture. ACS Appl Mater Interfaces, 2015, 7: 27035-27039

30 Wang H, Agarwal P, Zhao S, et al. Hyaluronic acid-decorated dual responsive nanoparticles of Pluronic F127, PLGA, and chitosan for targeted co-delivery of doxorubicin and irinotecan to eliminate cancer stem-like cells. Biomaterials, 2015, 72: 74-89

31 Lee H, Choi CH, Abbaspourrad A, et al. Encapsulation and enhanced retention of fragrance in polymer microcapsules. ACS Appl Mater Interfaces, 2016, 8: 4007-4013

32 Xia Y, Ribeiro PF, Pack DW. Controlled protein release from monodisperse biodegradable double-wall microspheres of controllable shell thickness. J Control Release, 2013, 172: 707-714

33 Cao J, Guenther RH, Sit TL, et al. Loading and release mechanism of red clover necrotic mosaic virus derived plant viral nanoparticles for drug delivery of doxorubicin. Small, 2014, 10: 5126-5136

34 Fu F, Shang L, Zheng F, et al. Cells cultured on core-shell photonic crystal barcodes for drug screening. ACS Appl Mater Interfaces, 2016, 8: 13840-13848

35 Liu X, Wang S. Three-dimensional nano-biointerface as a new platform for guiding cell fate. Chem Soc Rev, 2014, 43: 2385-2401

36 Suzuki H, Bae YH. Evaluation of drug penetration with cationic micelles and their penetration mechanism using an in vitro tumor model. Biomaterials, 2016, 98: 120-130

37 Liu L, Wu Q, Ma X, et al. Camptothecine encapsulated composite drug delivery system for colorectal peritoneal carcinomatosis therapy: biodegradable microsphere in thermosensitive hydrogel. Colloid Surf B-Biointerfaces, 2013, 106: 93-101

38 Shamanna RA, Lu HM, Croteau DL, et al. Camptothecin targets WRN protein: mechanism and relevance in clinical breast cancer. Oncotarget, 2016, 7: 13269-13284

Acknowledgments This work was supported by the National Natural Science Foundation of China (21473029 and 51522302), the NSAF Foundation of China (U1530260), the National Science Foundation of Jiangsu (BK20140028), the Program for New Century Excellent Talents in University, and the Scientific Research Foundation of Southeast University. D Yan also thanks the Foundation of Jiangsu Cancer Hospital (ZN201609) and Beijing Medical Award Foundation (YJHYXKYJJ-433).

Author contributions Zhao Y conceived the idea and designed the experiments; Li Y, Yan D and Fu F carried out the experiments; Zhao Y and Li Y analyzed the data and wrote the manuscript; Liu Y, Zhang B, Wang J, Shang $\mathrm{L}$ and $\mathrm{Gu} \mathrm{Z}$ contributed to scientific discussion of the article.

Conflict of interest The authors declare that they have no conflict of interest.

Supplementary information Supporting data are available in the online version of the paper. 

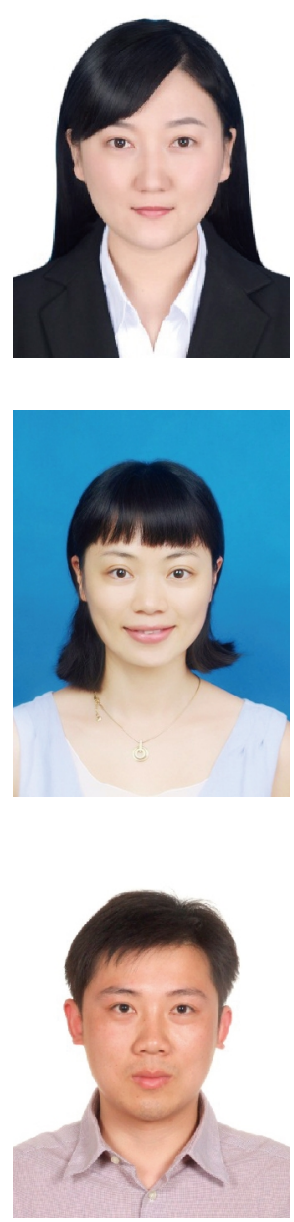

Yanna Li is currently a graduate student at the School of Biomedical Engineering, Southeast University. She joined Prof. Yuanjin Zhao's research group in 2013. Her current research focuses on the fabrication of microparticles by using droplet microfluidics.
Dan Yan is currently an associate chief physician at the Department of Pharmacy, Jiangsu Cancer Hospital. She received her PhD degree in 2011 from China Pharmaceutical University. Her current research focuses on the clinical drug delivery.

Yuanjin Zhao received his PhD degree in 2011 from Southeast University. In 2009-2010, he worked as a research scholar in Prof. David A. Weitz's group at the School of Engineering \& Applied Sciences, Harvard University. Since 2015, he has been a full professor of Southeast University. His current scientific interests include microfluidic-based materials fabrication, biosensors, and bio-inspired photonic nanomaterials.

\section{基于乳液微流控技术的核壳结构复合微胶囊药物载体开发研究}

李艳娜 ${ }^{1 \dagger}$, 燕丹 ${ }^{2 \dagger}$, 付繁繁 ${ }^{1}$, 刘羽霄 ${ }^{1}, 弓^{\circ}$ 彬 $^{2}$, 王洁 ${ }^{1}$, 商珞然 ${ }^{1}$, 顾忠泽 ${ }^{1}$, 赵远锦 ${ }^{*}$

摘要 微胶囊在药物递送系统中具有重要的应用价值. 目前关于该领域的研究主要集中于开发新型微胶囊来提高药物递送系统的效率. 本 文提出了一种可协同运输和缓慢释放药物的微胶囊, 其由明胶甲基丙烯酸接枝共聚物 (GelMa)内核和聚乳酸羟基乙酸共聚物(PLGA)外壳 组成. 在微胶囊的制备过程中, 使用液滴微流控技术, 将溶有盐酸阿霉素(DOX)的GelMa水溶液和溶有喜树碱(CPT)的PLGA油溶液乳化成 均匀的双乳液模板, 通过紫外固化模板内核, 通过溶剂挥发固化模板壳层. 该过程避免了乳液的破损及包裏液的流出, 因此可显著提高药 物的包裹效率. 通过调节微流控的流速, 还可精确地调节微粒的尺寸和结构. 由于所制备的微胶囊内核和外壳都为固化状态, 其包裹的活 性药物只能随着载体材料的降解而缓慢释放出来, 这就避免了其他种类药物载体所面临的药物突释现象. 本研究所开发的微胶囊的这些 优良特性使其成为药物递送系统中的理想选择. 